\title{
THE EFFECT OF FREE AND EXEMPLAR SORTING STRATEGIES \\ ON THE PERCEPTION OF VISUAL STRUCTURE \\ FOUND IN NONOBJECTIVE PAINTINGS
}

\section{Anna Kindler}

\section{Introduction}

The purpose of this study was to examine the effects of exemplar and free sorting strategies on naive subjects' perceptions of visual structure found in colored slides of nonobjective paintings.

Recent psychological studies concerned with the categories that mind uses to discriminate and qualify experience of perceiving art have been conducted using various methods of collecting similarity judgments data, ranging from paired comparisons to various sorting tasks.

The present study was designed to investigate the influence of method (namely different sorting tasks) on categorical perception and its objectives were based on the following reasoning. We felt, that the exemplar sort task, that involves a directed cognition experience (vs. a discovery cognition in the case of a free sort task), facilitates the category formation and that the subjects involved in an exemplar sort task should thus perform better than the subjects involved in a free sort task. Providing the exemplars for the subjects in the exemplar sort group supplies them with valuable cues about the characteristics of visual structure of a given category and allows them more confident classifications. We also felt that presenting subjects with examples best defining the visual structure of each category would help them in establishing the hierarchy within each category according to the degree to which its members exemplify its visual structure. 
We also predicted that among the various categories of visual structure the significance of cues that they contain may not be equal in all cases. Some categories are expected to be more salient than others and in both free and exemplar sorting conditions the subjects are expected to perform better on tasks involving categories that contain stronger, more salient cues. The difference between the groups involved in the two experimental conditions is expected to be the most significant on tasks involving nonsalient categories since the subjects in the exemplar sort group can benefit even more in this case from seeing the exemplars and can use this learning experience in their classification judgments.

\section{Method}

\section{Subjects}

Twenty-eight students not majoring in art were randomly assigned to two experimental conditions.

Exemplar sort group Free sort group
$N=14$
$N=14$

\section{Stimuli}

Thirty slides of nonobjective paintings were selected by judges from a set of 200 slides on nonobjective paintings. The choice of nonobjective paintings was influenced by the presence of direct cues (such as shape, color, line, etc.), and relative absence of distracting cues irrelevant to the visual structure. The slides were selected in such a way that they represented 6 different categories of visual structure. Each category consisted of 5 slides which were ranked by expert judges in terms of their being a good representation of a category (with the slide most clearly exemplifying the category being assigned 1 and so on).

Categories

6
Slides in each category

5 


\section{Procedure}

The subjects in the exemplar sort group were presented with an array of 24 slides randomly organized on a lighted display board. To the right of the array there was a row of 6 slides, the best examples of each category of visual structure as defined by experts. The subjects were asked to complete each category by moving the slides from the array and placing them under the exemplar slide of appropriate category. The subjects were instructed that each category should consist of a total of 5 slides.

After the subjects reported completion of the task they were asked to rank slides within each category, so the slide assigned 1 would be, in their opinion, the most representational for a given category and the one assigned 5 the least representational for this category.

The subjects in the free sort condition were presented with 30 slides randomly organized on a lighted display board and were asked to assign them to any desired number of categories according to their similarity in visual structure. Each category was required to consist of at least 3 slides. After the subjects completed the categorization task they were asked to rank each slide within each category (this part of the task was identical for subjects in both experimental conditions).

Each subject was tested individually. After the completion of the entire task the experimenter recorded subjects' judgments on a data collection sheet.

\section{Predictions of the Study}

A) The number of correctly matched slides will be significantly different for subjects in the exemplar and the free sort group. Variables: number of matches

Ho:

$$
\sum_{1}^{6} \# \text { match exam } \sum_{1}^{6} \# \text { match free }
$$


B) Ranking within each category will be significantly different for subjects in the exemplar sort group and the free sort group. Distance scores (defined as squared difference between experts' ranking of a slide and subjects' ranking of it), as well as total distance scores (prms), are expected to be significantly higher for subjects in the free sort condition than for the subjects in the exemplar sort group.

Variables: distance $=(\text { expert's rank - subject's rank })^{2}$

$$
\text { prms }=\sqrt{\text { dist }}=(4-\# \text { match })^{5}
$$

Ho: $\quad \sum_{1}^{6} \frac{\text { group }}{\text { prms exam }}=\sum_{1}^{6} \overline{\text { prms free }}$

C) It is expected that some categories will be more salient than others and that the performance on tasks involving these categories will be improved as compared to nonsalient categories by subjects in both experimental conditions. The average number of correctly matched slides will be higher and the average total distance score will be lower than in nonsalient categories.

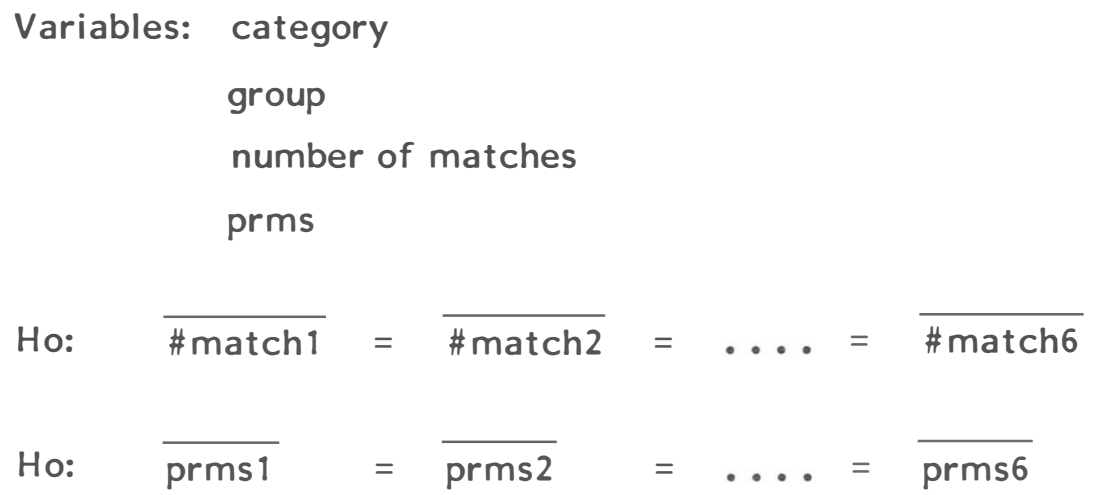

D) The difference between the groups is expected to be the most significant in the case of nonsalient categories. The subjects in the exemplar sort group will have a significantly higher average number of correctly matched slides and significantly lower average total distance score than the subjects in the free sort group on tasks involving nonsalient categories. 
$\begin{array}{ll}\text { Variables: } & \text { category } \\ & \text { group } \\ & \text { number of matches } \\ & \text { prms } \\ \text { Ho: } & \quad \frac{\text { \#match1exam }}{\text { \#match1free }}=\overline{\# \text { match2exam }}=. . . \text { \#match6free }\end{array}$

\section{Results}

A) A multivariate analysis of variance revealed that, as expected, the average number of correctly matched slides (20.5 in the free sort group and 21.5 in the exemplar sort group) was significantly higher in the exemplar sort group (Wilk's criterion $\underline{F}(6,21)=3.38, \underline{p}$ <.017). As expected, the subjects in the exemplar sort group tended to commit fewer mistakes in their categorization judgments than the subjects in the free sort group.

B) There was a significant difference between the groups in subjects' performance on the ranking task. As predicted, the subjects in the exemplar sort condition committed fewer mistakes in their ranking over all categories $(\overline{\text { prms free }}=35, \overline{\text { prms exam }}=27.5)$ and the multivariate analysis of variance has revealed a statistically significant group effect (Wilk's criterion $\underline{F}(6,21)=4.25, \underline{p}<$ $.006)$.

C) The analysis of the results has also confirmed the hypothesis that subjects' responses varied on tasks involving different categories. the category effect has proved to be statistically significant: both in terms of number of correctly matched slides (MANOVA Wilk's criterion $\underline{F}(5,22)=6.65, \underline{p}<.001)$ and in terms of ranking (MANOVA Wilk's criterion $F(5,21)=6.50, p<.001$ ).

The categories 2 and 3 (with the average number of matches in the free sort group 3.71 and 3.83, and in the exemplar sort group 3.85 and 3.78 , respectively) had the highest number of matches and the average number of matches for subjects in both groups combined for catagories 
2 and 3 was significantly different from the means of other categories $(p<.05$ and $p<.01$, respectively). (It was possible to analyze the results for both groups combined at this point since MANOVA has not revealed any significant difference between the groups in subjects' responses in categories 1 and 2). (See Figure 1).

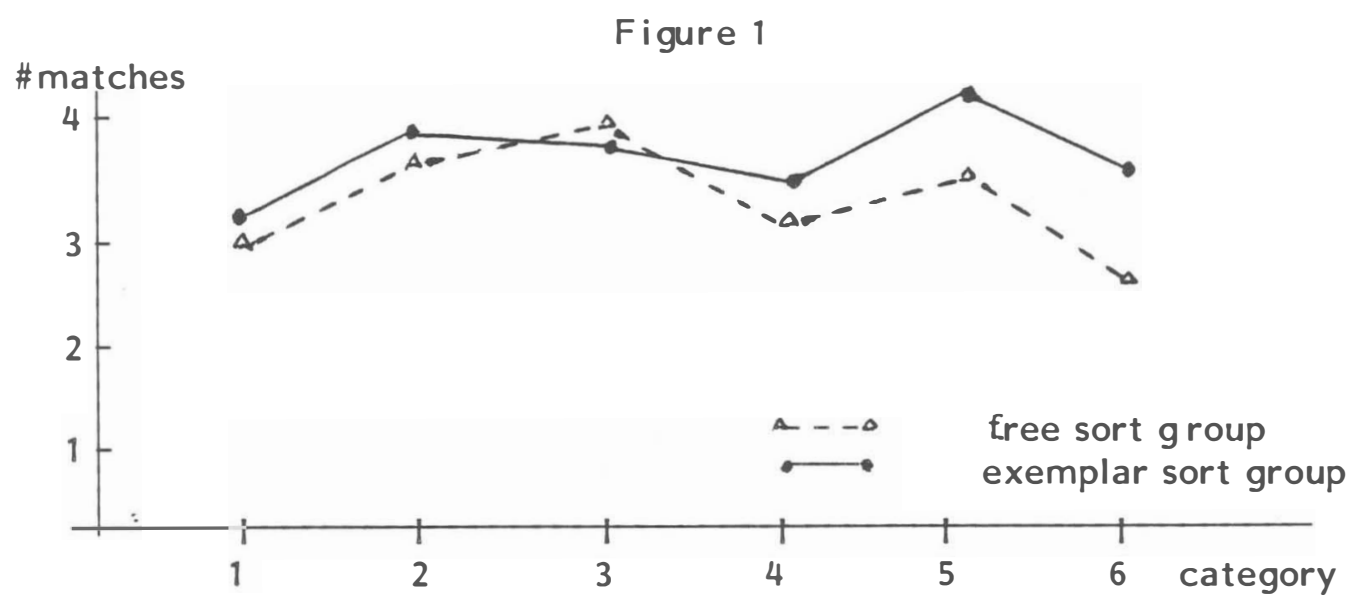

A comparison between the groups in terms of average number of matches with regard to specific categories.

Figure 2

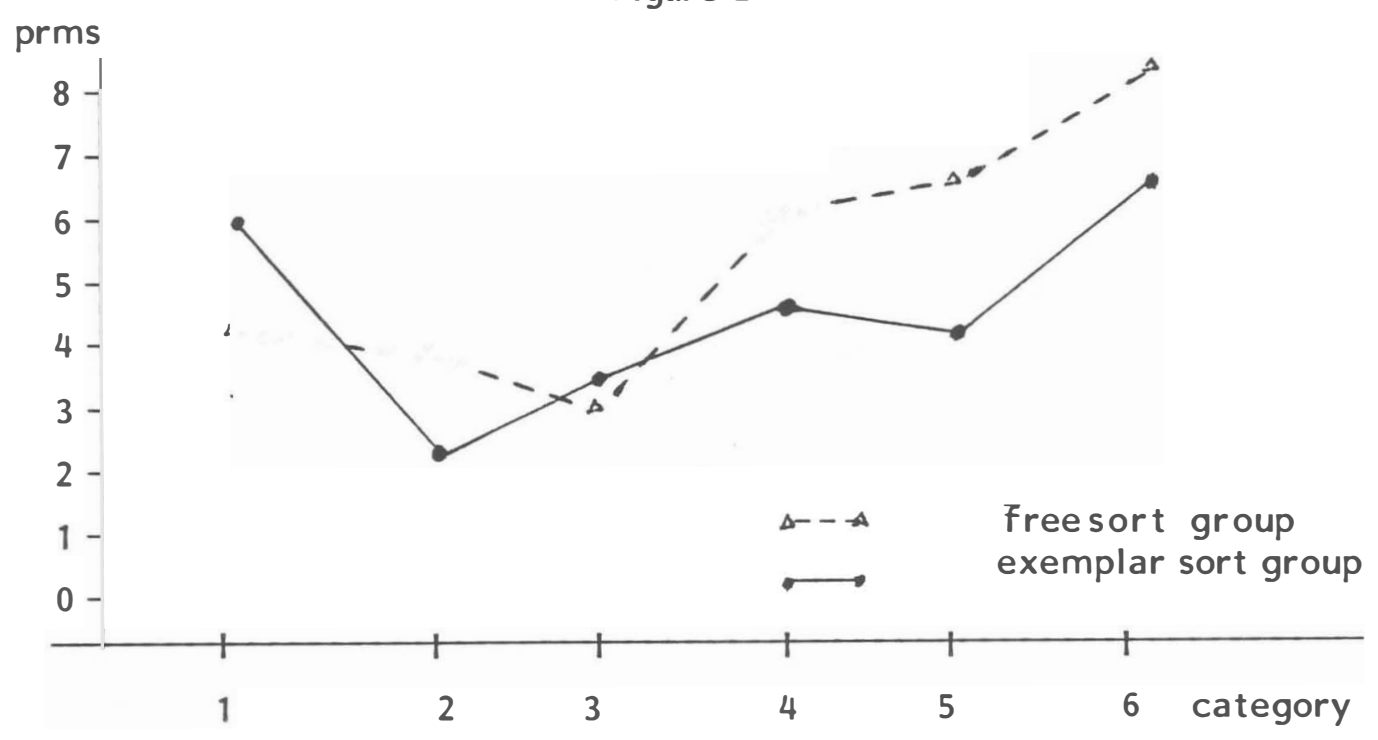

A comparison between the groups in terms of average total distance scores (prms) with regard to specific categories. 
This corresponds to the finding that the average total distance scores for slides in categories 2 and 3 (prms2exam $=2.43$, prms 3 exam $=3.67$ and prms 2 free $=3.98$, prms 3 free $=3.24$ ) were the lowest obtained and that for the subjects in both groups combined they were significantly lower than the means obtained in other categories $(p<.012$ and $p<$ .007 , respectively). (See Figure 2).

In tasks involving slides from category 6 the subjects in both groups tended to commit more mistakes: Both in terms of matching and ranking. (See Figures 1 and 2). The average number of matches for subjects in both groups in the category 6 (free sort group: 2.46 and the exemplar sort group: 3.21) was significantly lower $(p<.001)$ and the average total distance score $\overline{\text { prms6free }}=8.62$ and $\overline{\text { prms6exam }}=$ 5.83) was significantly higher $(p<.001)$ than the respective values in other categories. Even though in the category 5 the average number of matches for subjects in both groups has failed to be statistically significantly different from the means of other categories ( $p>$.095) it was possible to see that the average number of matches (especially in the case of the free sort group) was lower than in most other categories. Also, in terms of ranking the number of errors committed by subjects tended to be higher than in tasks involving other categories (See Figures 1 and 2). The difference, however, failed to prove statistically significant $(p>.052)$.

D) The multivarite analysis of variance has also indicated a significant group $x$ category interaction: both in terms of categorization and ranking tests $F(5,22)=4.24, \underline{p}<.007$ and $\underline{F}<$ $5,22)=5.15, \underline{p}<.003$, respectively). As predicted, the largest difference between the groups has occurred on tasks involving the categories identified as nonsalient (categories 5 and 6 ). For the category 5 the average number of correctly matched slides in the free sort group (3.42) was significantly lower $(p<.04)$ than the average number of matches in the exemplar sort group (3.85). Similarly, the average total distance score was significantly higher $(p<.01)$ for 
the subjects in the free sort group (6.27) as compared to the exemplar sort group (3.63). A noticeable difference between the free and the exemplar sort groups was also evident in tasks involving category 6. The average number of matches and the average total scores have not proved, however, to be statistically significantly different for the two groups ( $p>.09$ and $p>.08$, respectively).

Note: The exemplar slides have been excluded from the analysis to compensate for the fact that subjects in the exemplar sort group had them always positioned on the top of each category, and even though they were not instructed that the exemplar slides cannot be moved within each category, we felt that their inclusion in the analysis might artifically increase the difference between the groups.

\section{Discussion}

Overall, the results support the hypothesis that categorization strategies influence subjects' perceptions of visual structure. The cues contained in the exemplar sort task facilitate both category formation and ranking within the categories. It has also been shown that among the six categories of visual structure it is possible to identify some that are more salient than others and that classification and ranking in these categories is facilitated for subjects in both experimental conditions. The results also indicate that in the case of nonsalient categories, or the categories that contain less clear cues defining the visual structure, the exemplar sort task can significantly improve subjects' performance on both category formation and ranking tasks. 